\title{
RING 22 SYNDROME AND POLYEMBOLOKOILOMANIA
}

A 24 year-old severely retarded, hyperactive woman with a long history of foul smelling discharge from the nasal fossae, requiring removal under general anesthesia of foreign material (rocks, crayon, cotton, paper) on 9 separate occasions by an ENT surgeon, is reported from my own practice records in Chicago. The cumbersome term, polyembolokoilomania, has been applied to this behaviorism. The patient was referred from a residential home center where she was placed at 16 years because of violent and destructive behavior, eccentric habits, insomnia, refusal to eat, and self-injurious behavior, including body bruising and insertion of foreign bodies in her nose. She had occasional minor seizures. Her IQ was $<20$ on the Stanford-Binet. She did not speak but giggled frequently, often had a blank stare, and sometimes appeared catatonic. Her facial features were not dysmorphic. Attention to a visual object was poor. Hearing was intact. Her gait was uncoordinated, she was hypotonic and could not hop, but she had learned to swim. Deep tendon reflexes were hypoactive and plantar responses flexor. EEG showed a 8-9 $\mathrm{Hz} / \mathrm{sec}$ basic rhythm, excess theta and low-voltage-fast activity, but no epileptiform discharges. CT scan was normal. Birth was normal, she walked late at 2 years, and never talked intelligibly. An older brother was normal; a paternal aunt was retarded. She had a febrile seizure at 2 years and minor partial seizures controlled with phenytoin up to 9 years. EEGs had shown bilateral independent spikes in temporoparietal areas. Chromosome examination revealed $46, \mathrm{XX}, \mathrm{r}(22)$, with ring 22 chromosome present in all cells. Fragile $X$ was absent. Improvement followed behavioral modification and use of signs for communication. (Millichap JG. Ring 22 syndrome and selfinjurious behavior. Ped Neur Briefs Feb 1994; $\underline{8}: 10$ ).

COMMENT. Syndrome-specific behavioral phenotypes receive increasing attention in the literature, and the proposal that certain stereotypic behaviors are pathognomonic of a distinct syndrome requires further examination. The above case report is presented to demonstrate that one form of self-injurious behavior, involving repeated insertion of foreign bodies in the nose or other bodily orifices, may be common to ring 22 syndrome and the $17 \mathrm{p} 11.2$ deletion syndrome of Smith-Magenis.

Ring 22 syndrome is characterized by severe mental retardation, often associated with muscular hypotonia, poor coordination, hyperactivity and aggressive behavior, and rarely seizures. Facial features are not consistently dysmorphic but may include epicanthal folds, bushy eye-brows and long lashes, broad nasal bridge, higharched palate, clinodactyly and syndactyly, More than two thirds are female. The above case report emphasizes the indication for chromosomal analyses in girls with severe mental retardation and selfinjurious behavior, despite the absence of facial dysmorphism.

\section{RING CHROMOSOME 20 AND COMPLEX PARTIAL SEIZURES}

A ring chromosome 20 mosaicism in an 11-year-old girl with complex partial seizures resistant to medication is reported from the Department of Paediatric Neurology, University Hospital of Turku, Finland. Early psychomotor development was normal, but speech was delayed. She attended special schools for dyslexia, writing difficulties, and a wide range of cognitive problems, which became progressively worse. She had no dysmorphic features. Her behavior was infantile and interrupted by uncontrolled bursts of 
laughter. Epilepsy began with absence attacks at 7 years of age. A prolonged partial status at 10 years was associated with fronto-temporal focal seizure activity and bilateral spikes on the EEG, compatible with partial, secondarily generalized epilepsy. MRI was normal. Control of absence attacks with valproate was incomplete, and trials of oxcarbazepine and clobazam for complex partial seizures were only partially effective. The karyotype was mosaic 46,XX/46,XX,r(20)(p13q13). (Holopainen I et al. Ring chromosome 20 mosaicism in a girl with complex partial seizures. Dev Med Child Neurol Jan 1994;36:70-83). (Respond: Irma Holopainen MD, PhD, Department of Paediatric Neurology, University Hospital of Turku, Kiinamyllynk, 1-3, SF-20500 Turku, Finland).

COMMENT. Chromosome analysis may be indicated in a child with drug refractory idiopathic epilepsy and learning disabilities, despite the absence of dysmorphic features or other congenital anomalies.

Ring chromosome 20 syndrome is characterized by progressive cognitive impairments, behavior disorders and epilepsy resistant to conventional medications. Trials of newer antiepileptic medications with specificity against partial seizures (eg gabapentin) should provide more complete seizure control. (US Gabapentin Study Group No 5.

Gabapentin as add-on therapy in refractory partial epilepsy: A doubleblind, placebo-controlled, parallel-group study. Neurology Nov 1993; 43:2292-2298). Unlike many antiepileptic agents, gabapentin does not affect serum concentrations of AEDs and may be used as concurrent therapy. It had no hematologic, hepatic, pancreatic, or hypersensitivity adverse effects in this 12-week study of 306 patients.

\section{GOLDBERG-SHPRINTZEN SYNDROME \& CEREBRAL DYSGENESIS}

A 5-year-old girl with Goldberg-Shprintzen syndrome and an abnormal CT scan suggesting neuronal migration defect or brain dysgenesis is reported from the Departments of Pediatrics, Asahikawa Habilitation Center, and Kitami Red Cross Hospital, Japan. Hirschsprung disease was diagnosed at age 4 days, and congenital heart disease with heart failure at 3 months. Clonic convulsions developed at 5 years. Motor development was severely delayed; she sat at 15 months and was unable to stand at 5 years. She had microcephaly, hypertelorism, broad nasal bridge, high-arched palate, thick eyebrows, and an IQ of 25. CT showed frontal and temporal lobe atrophy. The clinical findings in 8 additional patients are summarized from published reports. No chromosomal abnormalities were detected. (Tanaka $\mathrm{H}$ et al. Hirschprung disease, unusual face, mental retardation, epilepsy, and congenital heart disease: GoldbergShprintzen syndrome. Pediatr Neurol Nov/Dec 1993;9:479-81). (Respond: Dr Tanaka, Department of Pediatrics, Asahikawa Habilitation Center for Disabled Children, Shunkodai 2-1, Asahikawa 078, Japan).

COMMENT. Waardenburg (congenital deafness, white forelock and depigmented, joined eyebrows, heterochromia iridum, broad nasal bridge) and Smith-Lemli-Opitz (micrognathia, microcephaly, retardation, broad nose and anteverted nostrils, skeletal and urogenital abnormalities) syndromes have similar facial features to those of G-P syndrome and are sometimes complicated by Hirschprung disease. 